\title{
Application of artificial neural network on vibration test data for damage identification in bridge girder
}

\author{
S. J. S. Hakim and H. Abdul Razak \\ ${ }^{1}$ Department of Civil Engineering, University of Malaya, Kuala Lumpur 50603, Malaysia.
}

Accepted 11 October, 2011

\begin{abstract}
Structures are exposed to damage during their service life which can severely affect their safety and functionality. Thus, it is important to monitor structures for the occurrence, location and extent of damage. Artificial neural networks (ANNs) as a numerical technique have been applied increasingly for damage identification with varied success. ANNs are inspired by human biological neurons and have been used to model some specific problems in many areas of engineering and science to achieve reasonable results. ANNs have the ability to learn from examples and then adapt to changing situations when sufficient input-output data are available. This paper presents the application of ANNs for detection of damage in a steel girder bridge using natural frequencies as dynamic parameters. Dynamic parameters are easy to implement for damage assessment and can be directly linked to the topology of structure. In this study, the required data for the ANNs in the form of natural frequencies will be obtained from experimental modal analysis. This paper also highlights the concept of ANNs followed by the detail presentation of the experimental modal analysis for natural frequencies extraction.
\end{abstract}

Key words: Artificial neural networks (ANNs), back propagation (BP), damage identification, natural frequency.

\section{INTRODUCTION}

Damage can be defined as a weakening of the structure that adversely affects its current or future performance which may cause undesirable displacements, stresses or vibrations to the structure. Therefore, one of the most important requirements in the evaluation of existing structural systems and ensuring a safe performance during their service life is damage identification.

Many techniques have been applied to identify damage in civil structures. Visual inspections have always been the most common approaches used in detecting damage on a structure (Abdul and Choi, 2001). These methods are time consuming and cannot indicate that a structure is fault-free without testing the entire structure in detail. Furthermore, if damage is buried deep within the structure it may not be visible or detectable by these localized techniques.

*Corresponding author. E-mail: jamalhakim@siswa.um.edu.my. Tel: 0060123551940.
Among the structure identification methods that have been applied dramatically in recent years are artificial neural networks (ANNs) due to its powerful computational and excellent pattern recognition ability for detecting damage in structural engineering. Once ANNs are trained, they are capable of pattern recognition and classification, and are robust in the presence of noise. These characteristics make ANNs powerful tools for vibrational damage identification. By incorporating ANNs, accuracy and reliability of damage identification can be improved.

Several authors have applied modal parameters as inputs of ANNs in structural damage identification. For example, these studies (Suh et al., 2000; Jeyasehar and Sumangala, 2006; Kim and Kapania, 2002; Kirkegaard and Rytter, 1994) applied ANN for predicting damage using natural frequencies. ANNs have been applied by Lee et al. (2005) to identify damage in bridges using mode shape. Also, Sahin and Shenoi (2003) presented a damage assessment algorithm using a combination of changes in natural frequencies and curvature mode shapes 


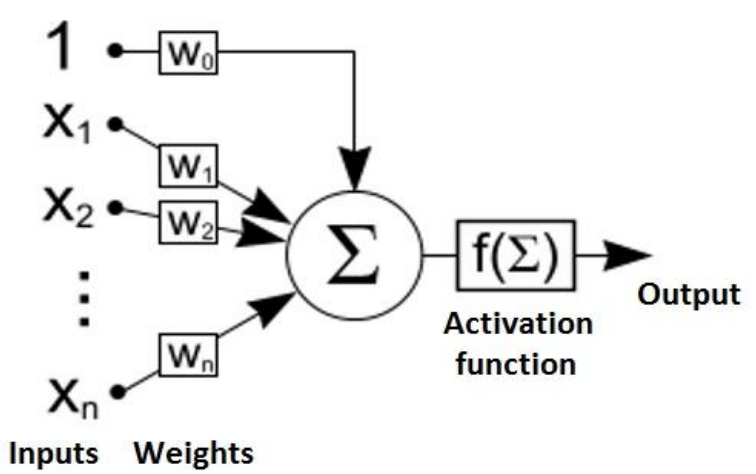

Figure 1. Schematic structure of an artificial neuron.

as input in ANNs for the location and severity prediction of damage in numerical models of composite beam structures.

The main focus of this research is to investigate the feasibility of applying an ANN trained with only natural frequency data to identify the damage in steel bridge girder structure. Natural frequencies of a structure have strong effect on damage and are applied as effective input parameters to train the ANN in the present study.

The required data for the ANNs in form of natural frequencies are obtained from experimental modal analysis. For this study, it was shown that an ANN trained only with natural frequency data can determine the severity of damage with less than $6 \%$ error, and seems to be quite promising in terms of accuracy.

\section{ARTIFICIAL NEURAL NETWORKS (ANNS)}

ANNs are simplified models of the human brain and evolved as one of the most useful mathematical concepts used in almost all branches of science and engineering. They have the ability to learn from experience in order to improve their performance and to adapt themselves to changes in the environment (Hola and Schabowicz, 2005; Mansour et al., 2004). ANNs can provide meaningful answers even when the data to be processed include errors or are incomplete and can process information extremely rapidly when applied to solve real problems.

ANN is composed of several processing elements, namely, neurons that are interconnected with each other. Figure 1 shows the model of an artificial neuron which consists of a neuron that receives weighted inputs (w) that are summed and passed through an activation function (f) to produce a single output.

A typical neural network has three layers, namely, the input layer, the hidden layer and the output layer. Signals are received at the input layer, pass through the hidden layer and reach the output layer. Each layer can have a different number of neurons and activation functions, such as sigmoid and linear functions. All neurons are

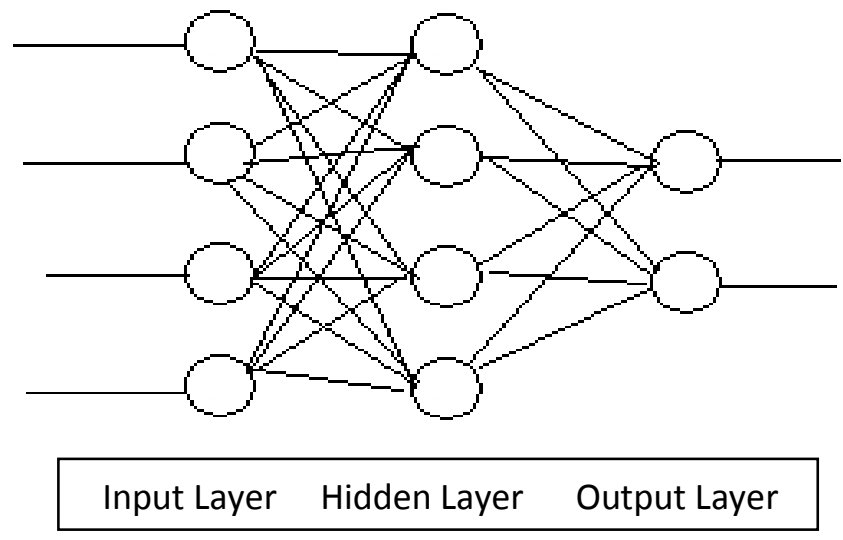

Figure 2. Architecture of a typical multilayer feedforward neural network.

interconnected to the neurons in the next layer through their weights. Architecture of an ANN with 4 neurons in input layer, 4 neurons in hidden layer and 2 neurons in output layer is demonstrated as shown in Figure 2.

Backpropagation algorithm in multi-layer feed-forward supervised networks is considered to be the most applicable, due to the mathematical design of the learning complex nonlinear relationships (Fonseca and Vellasco, 2003). This algorithm has a performance index, which is the least mean square error (MSE) (Hakim et al., 2011; Ince, 2004; Lee, 2003; Rumelhart et al., 1986). In MSE algorithm, the error is calculated as the difference between the target output and the network output. Among various neural networks, multi-layer perceptron (MLP) is most commonly used in structural identification problems. Their applications to engineering problems have been summarized and reported in literature (Chen et al., 1995; Ghaboussi and Joghatie, 1995; Wu et al, 2002; Xu et al., 2002).

\section{DAMAGE DETECTION STRATEGY AND EXPERIMENTAL MODAL ANALYSIS}

When there is damage in a structure, the stiffness in general will reduce. Since the natural frequencies of a structure depend on stiffness, the natural frequencies will also reduce when there is damage. As mentioned earlier, the aim of this research is to find out the severity of damage in a steel bridge girder using ANNs and experimental modal analysis. Therefore, this work proposed to apply the first five natural frequencies as inputs of ANN for prediction of damage severity. To identify the natural frequencies as dynamic properties of the bridge girder, experimental modal analysis was performed with different damage scenarios.

In the first stage, modal testing was performed using an undamaged bridge girder in order to obtain the modal frequencies. Later, numerous damage scenarios were 


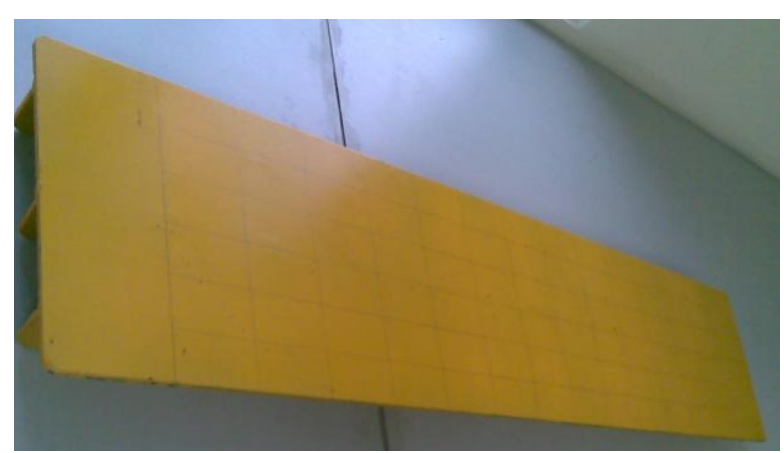

A

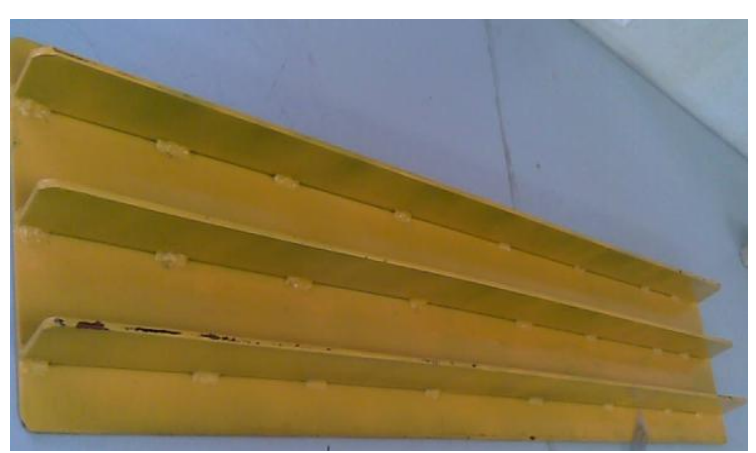

B

Figure 3. Test structure.

Table 1. Frequencies of the first five modes at undamaged state.

\begin{tabular}{ccccc}
\hline \multicolumn{5}{c}{ Natural frequencies $(\mathrm{Hz})$} \\
\hline Mode 1 & Mode 2 & Mode 3 & Mode 4 & Mode 5 \\
\hline 110.41 & 177.2 & 352.5 & 428.05 & 701.4 \\
\hline
\end{tabular}

Table 2. Cross-section loss of the second moment of area (I) with different damage severity.

\begin{tabular}{cccc}
\hline $\begin{array}{c}\text { Damage severity } \\
(\mathbf{m m})\end{array}$ & $\begin{array}{c}\text { Cross-section loss of the second } \\
\text { moment of area }(\mathbf{I})(\%)\end{array}$ & $\begin{array}{c}\text { Damage severity } \\
(\mathbf{m m})\end{array}$ & $\begin{array}{c}\text { Cross-section loss of the second } \\
\text { moment of area }(\mathbf{I})(\%)\end{array}$ \\
\hline 2 & 11.5 & 18 & 73.78 \\
4 & $22.10 \%$ & 20 & 78.40 \\
6 & 31.85 & 22 & 82.44 \\
8 & 40.73 & 24 & 85.94 \\
10 & 48.80 & 26 & 88.94 \\
12 & 56.10 & 28 & 91.48 \\
14 & 62.67 & 30 & 93.60 \\
16 & 68.55 & - & - \\
\hline
\end{tabular}

created by introducing different severity of damage at different locations along the bridge girder.

The results of experimental modal analysis will be use as training data for the ANN algorithm. By incorporating the training data, ANN will be able to give outputs in terms of damage severity using the five first natural frequencies.

The test structure as shown in Figure 3 was fabricated from a plate with the dimensions of $1200 \mathrm{~mm}$ length including a $100 \mathrm{~mm}$ overhang at both support ends and 210 and $5 \mathrm{~mm}$ in width and height, respectively. Three stiffeners as shown in Figure $3 \mathrm{~B}$ were fixed along the length of plate with dimensions of $1200 \times 50 \times 5 \mathrm{~mm}$ in length, width and height, respectively. The modulus of elasticity of the steel, the Poisson's ratio and the density were, $2.1 \times 10^{11}, 0.2$ and $7,850 \mathrm{~kg} / \mathrm{m}^{3}$, respectively.

The test structure was tested in its undamaged state and under different damaged states to determine the first five natural frequencies. These natural frequencies were identified from the frequency response functions (FRF) measurements as peaks. Table 1 lists the first five natural frequencies for the undamaged bridge girder.

In the experimental study, various damage scenarios were given to the test structure. These scenarios consisted of seven locations with fifteen severities for each location. The seven damage locations were at $\mathrm{L} / 13,2 \mathrm{~L} / 13,3 \mathrm{~L} / 13,4 \mathrm{~L} / 13,5 \mathrm{~L} / 13,6 \mathrm{~L} / 13$ and $\mathrm{L} / 2$ of the span length. Only one half of the test structure was used as damage model due to symmetry. Damage was gradually induced by a grinder to cut a slot from the soffit of the middle stiffener of the structure. These damage severities correspond to a cross-section loss of the second moment of area (I) as shown in Table 2.

For each damaged severity, five peaks were identified 
Table 3. Natural frequencies of the first five modes for damage at $4 \mathrm{~L} / 13$.

\begin{tabular}{cccccc}
\hline \multirow{2}{*}{ Damage severity $(\mathbf{m m})$} & \multicolumn{5}{c}{ Number of mode shape } \\
\cline { 2 - 6 } & $\mathbf{1}$ & $\mathbf{2}$ & $\mathbf{3}$ & $\mathbf{4}$ & $\mathbf{5}$ \\
\hline 2 & 109.01 & 176.11 & 348.07 & 429.41 & 699.79 \\
4 & 108.16 & 175.64 & 344.19 & 426.49 & 701.11 \\
6 & 107.37 & 172.18 & 342.86 & 426.73 & 700.76 \\
8 & 107.77 & 173.27 & 338.48 & 427.01 & 697.82 \\
10 & 106.93 & 170.55 & 337.50 & 425.60 & 697.11 \\
12 & 106.19 & 168.12 & 334.92 & 423.60 & 695.81 \\
14 & 105.58 & 167.54 & 332.10 & 421.54 & 692.44 \\
16 & 105.23 & 165.43 & 331.12 & 419.65 & 692.66 \\
18 & 104.05 & 163.87 & 332.43 & 418.32 & 693.12 \\
20 & 104.66 & 164.34 & 334.41 & 417.04 & 693.70 \\
22 & 103.44 & 164.68 & 332.84 & 415.65 & 693.02 \\
24 & 102.51 & 164.20 & 331.87 & 412.11 & 692.91 \\
26 & 102.67 & 163.83 & 332.11 & 410.76 & 691.54 \\
28 & 102.29 & 163.78 & 330.06 & 410.06 & 690.23 \\
30 & 102.02 & 163.36 & 330.41 & 408.44 & 689.21 \\
\hline
\end{tabular}

Table 4. Range of input parameters.

\begin{tabular}{lcc}
\hline Input parameter & Minimum $(\mathbf{H z})$ & Maximum $(\mathbf{H z})$ \\
\hline $1^{\text {st }}$ N.F & 98.35 & 110.87 \\
$2^{\text {nd }}$ N.F & 152.38 & 178.15 \\
$3^{\text {rd }}$ N.F & 319.28 & 353.44 \\
$4^{\text {th }}$ N.F & 400.54 & 430.61 \\
$5^{\text {th }}$ N.F & 670.33 & 701.4 \\
\hline
\end{tabular}

N.F: Natural frequency.

which were related to the modal frequencies of the structure. Table 3 shows the first five frequencies for a damage case for the test structure, and it is apparent that the natural frequencies drop when damage is induced. These results will be used for training ANNs for damage severity.

\section{DAMAGE DETECTION USING ANN}

The first step in ANNs is to prepare suitable and accurate data sets that can be used to train a network to recognize the pattern from the data set. Ideally, this data set should be the actual real life response of the structural model test results or through numerical modeling, or a combination of all these types of data sets (Chakraborty, 2005).

In this study, 203 different sets of data from undamaged and damaged of scaled down steel girder bridge deck were collected from the experimental modal analysis. These data were gathered for damage severity of the test structure containing the first five natural frequencies. Different neural network models were conducted, trained and tested using these available data. Out of the 203 data sets, only 139 were used as training data sets. The numbers of data sets for testing and the validation phase were 32 .

Division of the data was carried out randomly between the three sets. The ranges of input parameters are as shown in Table 4. The scattering of input information for the training phase will affect the accuracy of a neural network. Therefore, classification of the input information is very important in the training phase.

\section{Training of ANN}

The training of the ANN with appropriate data set is the process of changing the weights systematically in order to attain some desired results for a given set of inputs. The process of training is successfully completed, when the iterative process has converged.

Presenting the whole set of training samples to the network is called iteration, and the number of iterations means the number of times that the whole set of samples are presented to the network. In this study, feed forward back propagation algorithm for ANN training was selected. At first an input vector comprising of the first five natural frequencies was fed to the input layer. This input vector produces a set of output. The difference between the given output and the target output is error, which will propagate through the network in backward step.

During this process, the mean square error (MSE) will be minimized, and consequently, the output of ANN will be close to the target output. An accurate trained ANN 
Table 5. Damage index values as output of ANN.

\begin{tabular}{cccc}
\hline Damage severity $(\mathrm{mm})$ & Damage index & Damage severity $(\mathbf{m m})$ & Damage index \\
\hline 2 & 0.8850 & 18 & 0.2622 \\
4 & 0.7790 & 20 & 0.2160 \\
6 & 0.6815 & 22 & 0.1756 \\
8 & 0.5927 & 24 & 0.1406 \\
10 & 0.5520 & 26 & 0.1106 \\
12 & 0.4390 & 28 & 0.0852 \\
14 & 0.3733 & 30 & 0.0640 \\
16 & 0.3145 & - & - \\
\hline
\end{tabular}

gives successful predictions when a new sample is given as input. The output parameter of ANN is damage index, representing the severity of damage. This damage index is the ratio of cross-section loss of the second moment of area for damaged case to undamaged case. The value of damage index based on different damage severity is given in Table 5.

The network has been trained using the Alyuda Neuro Intelligence software, version 2.2. Once the network is trained using training data, it is ready for predicting the severity of damage in the test structure.

As mentioned previously, 139 training data sets were used for the training process of the network. These data were normalized between -1 and 1 , and were fed to the input neurons. The values of damage index corresponding to each set of natural frequencies have also been fed to the network as desired outputs. In this research study, the training process was stopped when any of the following conditions were satisfied:

1. The maximum number of iterations reached 70000 .

2. The mean square error (MSE) of the network for the training set reached 0.004 .

During the training of ANN, the best network with the optimum parameters such as, connectivity weights and biases, the numbers of hidden layers and the numbers of neurons in each layer, type of activation function in hidden and output layers will be determined. Also, the rate of learning and momentum value will be specified.

In this study, different architecture networks having different conditions were determined. Architecture specifications of 25 most important ANNs in order to obtain the final developed ANN architecture are tabulated in Table 6. It is obvious from this table that in architectures with one hidden layer, that is, networks $N_{1}$ to $\mathrm{N}_{15}$, MSE is lower than other networks with two hidden layers. Also, in architectures with one hidden layers, MSE is less than when there is two hidden layer.

After trying different networks with one and two hidden layers and taking into consideration the network error, one hidden layer in the architecture of ANN was decided in this study. However, in a network with one hidden layer, good convergence has been achieved and the back propagation in this research was limited to one hidden layer, which yields a total of three layers.

Thus, the networks $N_{16}$ to $N_{24}$ in Table 6 are not acceptable for the purpose of this work. The decision on how many hidden neurons should be used in hidden layer is rather arbitrary, and has been usually decided by trial and error. Table 6 shows the effect of different number of hidden neurons on MSE.

It can be seen, that with the increase of hidden neurons, training error is reduced, but there are a critical number of hidden neurons existing for minimizing error rate. The reason is that, with too many hidden neurons, a network can simply memorize the correct response to each pattern in its training set instead of learning a general solution.

So, in this study with increasing number of hidden neurons, MSE is decreased, but variations in MSE values for more than 15 neurons are significant. Also, it is apparent from this table that in networks $N_{10}$ and $N_{11}$, error is small, but numbers of hidden neurons and connectivity weights are high and computationally complicated and take longer time. For example networks $N_{10}$ and $N_{11}$ have 106 and 113 connectivity weights, respectively, with 70000 iterations.

It can be demonstrated in this table that networks $\mathrm{N}_{6}$ and $\mathrm{N}_{7}$, have acceptable error and small hidden neurons. Also, average training error is lower than other networks. Consequently, the correlation of these networks is higher than other networks. However, network $\mathrm{N}_{7}$ is selected as the best possible architecture in this study. It is important to note that utilizing more than 15 neurons in network makes the computation process complicated and expensive in terms of time. In summary, in order to have minimum compatibility cost and high accuracy, the number of hidden layer is fixed to 15 .

However, the network architecture decided in this study was 5-12-1. This architecture comprises of five neurons in the input layer corresponding to the five first natural frequencies, one hidden layer with twelve neurons and one neuron in the output layer corresponding to severity of damage in the test structure. The final architecture for this network is depicted in Figure 4. 
Table 6. Specifications of different ANN architectures.

\begin{tabular}{|c|c|c|c|c|c|c|c|}
\hline $\begin{array}{l}\text { No. of } \\
\text { network }\end{array}$ & Architecture & $\begin{array}{c}\text { No. of } \\
\text { weights }\end{array}$ & $\begin{array}{l}\text { Network error } \\
\text { (MSE) }\end{array}$ & $\begin{array}{c}\text { Average } \\
\text { training error }\end{array}$ & $\begin{array}{c}\text { Average } \\
\text { validation error }\end{array}$ & $\begin{array}{l}\text { Average testing } \\
\text { error }\end{array}$ & Correlation \\
\hline $\mathrm{N}_{1}$ & $5-6-1$ & 43 & 0.004917 & 0.062950 & 0.067014 & 0.056536 & 0.934458 \\
\hline $\mathrm{N}_{2}$ & $5-7-1$ & 50 & 0.004818 & 0.061358 & 0.060565 & 0.063842 & 0.968471 \\
\hline $\mathrm{N}_{3}$ & $5-8-1$ & 57 & 0.004687 & 0.059020 & 0.075203 & 0.062626 & 0.971163 \\
\hline $\mathrm{N}_{4}$ & $5-9-1$ & 64 & 0.004729 & 0.060415 & 0.070857 & 0.059195 & 0.965405 \\
\hline $\mathrm{N}_{5}$ & $5-10-1$ & 71 & 0.004911 & 0.065210 & 0.054702 & 0.065236 & 0.965412 \\
\hline $\mathrm{N}_{6}$ & $5-11-1$ & 78 & 0.004213 & 0.057827 & 0.062257 & 0.073811 & 0.970089 \\
\hline $\mathrm{N}_{7}$ & $5-12-1$ & 85 & 0.004046 & 0.056199 & 0.077919 & 0.062532 & 0.973051 \\
\hline $\mathrm{N}_{8}$ & $5-13-1$ & 92 & 0.004012 & 0.060152 & 0.060649 & 0.062061 & 0.966636 \\
\hline $\mathrm{N}_{9}$ & $5-14-1$ & 99 & 0.004911 & 0.059528 & 0.069692 & 0.060437 & 0.971859 \\
\hline$N_{10}$ & $5-15-1$ & 106 & 0.004316 & 0.057448 & 0.06793 & 0.066905 & 0.972319 \\
\hline$N_{11}$ & $5-16-1$ & 113 & 0.004301 & 0.056598 & 0.057525 & 0.060834 & 0.967632 \\
\hline $\mathrm{N}_{12}$ & $5-17-1$ & 120 & 0.004817 & 0.062765 & 0.061197 & 0.068115 & 0.968176 \\
\hline $\mathrm{N}_{13}$ & $5-18-1$ & 127 & 0.004781 & 0.059691 & 0.067138 & 0.055565 & 0.968839 \\
\hline $\mathrm{N}_{14}$ & $5-19-1$ & 134 & 0.005071 & 0.063384 & 0.052247 & 0.06726 & 0.967966 \\
\hline $\mathrm{N}_{15}$ & $5-20-1$ & 141 & 0.004824 & 0.059392 & 0.065052 & 0.064687 & 0.967888 \\
\hline $\mathrm{N}_{16}$ & $5-5-2-1$ & 45 & 0.005002 & 0.062950 & 0.067014 & 0.056536 & 0.934458 \\
\hline $\mathrm{N}_{17}$ & $5-5-3-1$ & 52 & 0.005187 & 0.064880 & 0.065875 & 0.067295 & 0.927854 \\
\hline $\mathrm{N}_{18}$ & $5-5-4-1$ & 59 & 0.004916 & 0.060600 & 0.063102 & 0.061959 & 0.937129 \\
\hline $\mathrm{N}_{19}$ & $5-5-5-1$ & 66 & 0.006179 & 0.071418 & 0.080273 & 0.086346 & 0.907112 \\
\hline $\mathrm{N}_{20}$ & $5-6-3-1$ & 61 & 0.005119 & 0.062688 & 0.067576 & 0.064606 & 0.930475 \\
\hline $\mathrm{N}_{21}$ & $5-6-6-1$ & 85 & 0.005814 & 0.068288 & 0.063493 & 0.064204 & 0.933829 \\
\hline $\mathrm{N}_{22}$ & $5-8-4-1$ & 89 & 0.004788 & 0.059434 & 0.076234 & 0.056695 & 0.935407 \\
\hline $\mathrm{N}_{23}$ & $5-10-5-1$ & 121 & 0.004615 & 0.059642 & 0.069583 & 0.055554 & 0.939118 \\
\hline $\mathrm{N}_{24}$ & $5-12-6-1$ & 157 & 0.007614 & 0.079762 & 0.070728 & 0.072397 & 0.891491 \\
\hline $\mathrm{N}_{25}$ & 5-12-8-1 & 185 & 0.007517 & 0.078607 & 0.073338 & 0.068069 & 0.911243 \\
\hline
\end{tabular}

Different combinations of momentum value and learning rate using trial and error have been executed for this architecture. Results showed that constructed ANN with learning rate 0.2 and 0.6 for momentum parameter, yields minimum error. Therefore, these values have been found to be most suitable for selected network in this study. In this network, Hyperbolic Tangent Sigmoid and LogSigmoid functions were applied in hidden and output layer as activation functions, respectively.

The network is then trained for 70,000 iterations until the training error reach minimum and the network is stable. In short, training of this architecture (5-12-1) with value of 0.2 and 0.6 for learning rate and momentum, respectively has been continued till 70,000 iterations and the average training error reaches $5.6 \%$. After that, the network was saved and the corresponding connectivity weights were saved. Comparison of predicted damage severity by ANN and actual value from experimental testing (target value) is depicted in Figure 5.

For more clarity, experimental and predicted values in training sets by considering MSE for new data sets are tabulated in Table 7. Calculation of average training error as demonstrated in Table 6 , shows that ANN predicted damage severity with average percentage error of $5.6 \%$ for training data sets.

As can be seen in Table 6, the selected network was capable in providing good correlation between the natural frequency information and extent of damage in terms of damage index for a given set of natural frequencies. This network has minimum error and maximum correlation, that is, 0.973 as compared to other networks according to Table 6.

\section{Neural network testing}

The testing set is used to visually inspect performance after training. After the network was trained, testing of ANN was carried out to avoid over-fitting and to assess the confidence in the performance of the trained network. In this step, the trained network is tested with data, which were not present in the training data set. After training, the network has learnt the samples and when tested with new data, it should be able to predict the severity of damage with an acceptable error. The testing process has been done using a total of 32 data sets.

Figure 6 shows the comparison between predicted damage severity and the experimental data, when the 


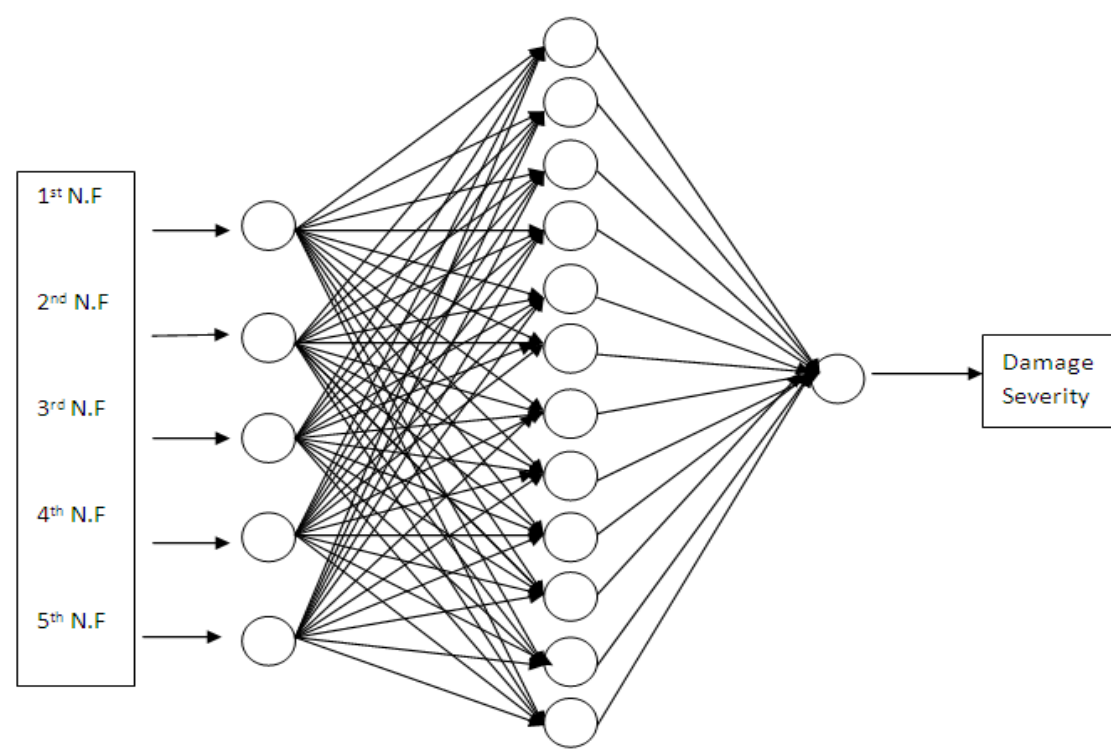

Figure 4. Final architecture of ANN.

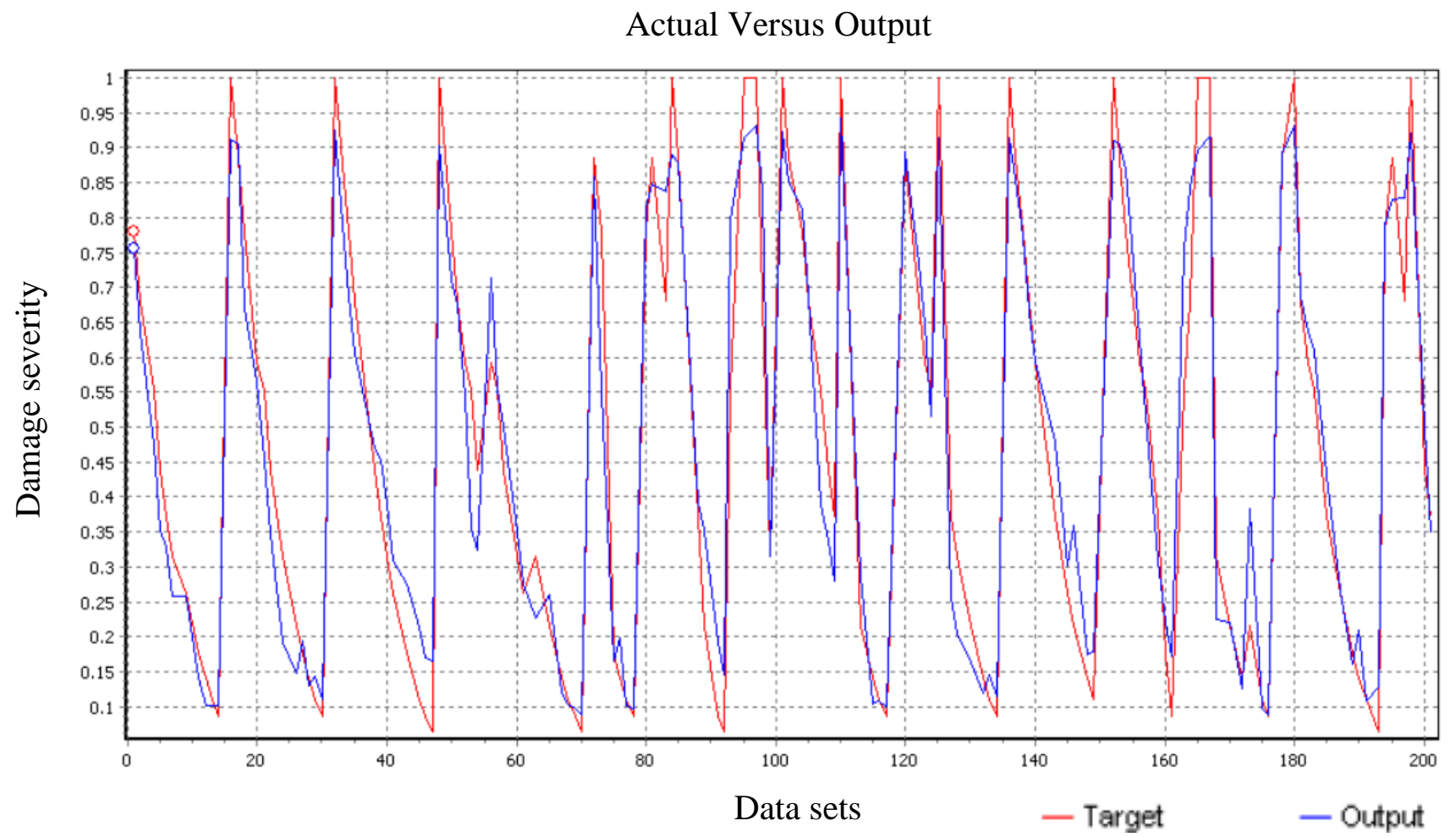

Figure 5. Comparison of damage severity predicted by ANN and experimental for training sets.

network has been constructed to predict 32 number of new data set. From this figure it can be highlighted that there is a good agreement between the predicted values and that of the experimental data.

ANN was successful in predicting the severity of damage with MSE of $6.2 \%$ for testing sets and was very close to actual output. However, in predicting the severity of damage, the network could not predict accurately for a few samples. This observation may be due to the fact that the ANN was not being provided with large number 
Table 7. Comparison between experimental and predicted values in training.

\begin{tabular}{ccccc}
\hline Data set & $\begin{array}{c}\text { Target value } \\
\text { (Experimental) }\end{array}$ & $\begin{array}{c}\text { Network value } \\
\text { (Predicted) }\end{array}$ & $\begin{array}{c}\text { Mean square } \\
\text { error }\end{array}$ & $\begin{array}{c}\text { Percentage of } \\
\text { error }\end{array}$ \\
\hline 1 & 0.779 & 0.756294 & 0.022706 & 2.91471 \\
2 & 0.6815 & 0.636513 & 0.044987 & 6.601113 \\
3 & 0.552 & 0.474069 & 0.077931 & 14.11802 \\
4 & 0.552 & 0.554102 & 0.002102 & 0.38077 \\
5 & 0.3733 & 0.327399 & 0.045901 & 12.29612 \\
6 & 1 & 0.914769 & 0.085231 & 8.523133 \\
7 & 0.2622 & 0.258852 & 0.003348 & 1.276875 \\
8 & 0.1756 & 0.14161 & 0.03399 & 19.35667 \\
9 & 0.1106 & 0.101941 & 0.008659 & 7.829482 \\
10 & 0.1106 & 0.102159 & 0.008441 & 7.632046 \\
11 & 0.0852 & 0.10055 & 0.01535 & 18.01664 \\
12 & 1 & 0.912039 & 0.087961 & 8.796062 \\
13 & 0.885 & 0.90436 & 0.01936 & 2.187516 \\
14 & 0.779 & 0.670656 & 0.108344 & 13.90805 \\
15 & 0.5927 & 0.565533 & 0.027167 & 4.583656 \\
16 & 0.552 & 0.453006 & 0.098994 & 17.93374 \\
17 & 0.439 & 0.348461 & 0.090539 & 20.62401 \\
18 & 1 & 0.92439 & 0.07561 & 7.56102 \\
19 & 0.216 & 0.147551 & 0.068449 & 31.68936 \\
20 & 0.1756 & 0.194219 & 0.018619 & 10.60321 \\
\hline
\end{tabular}

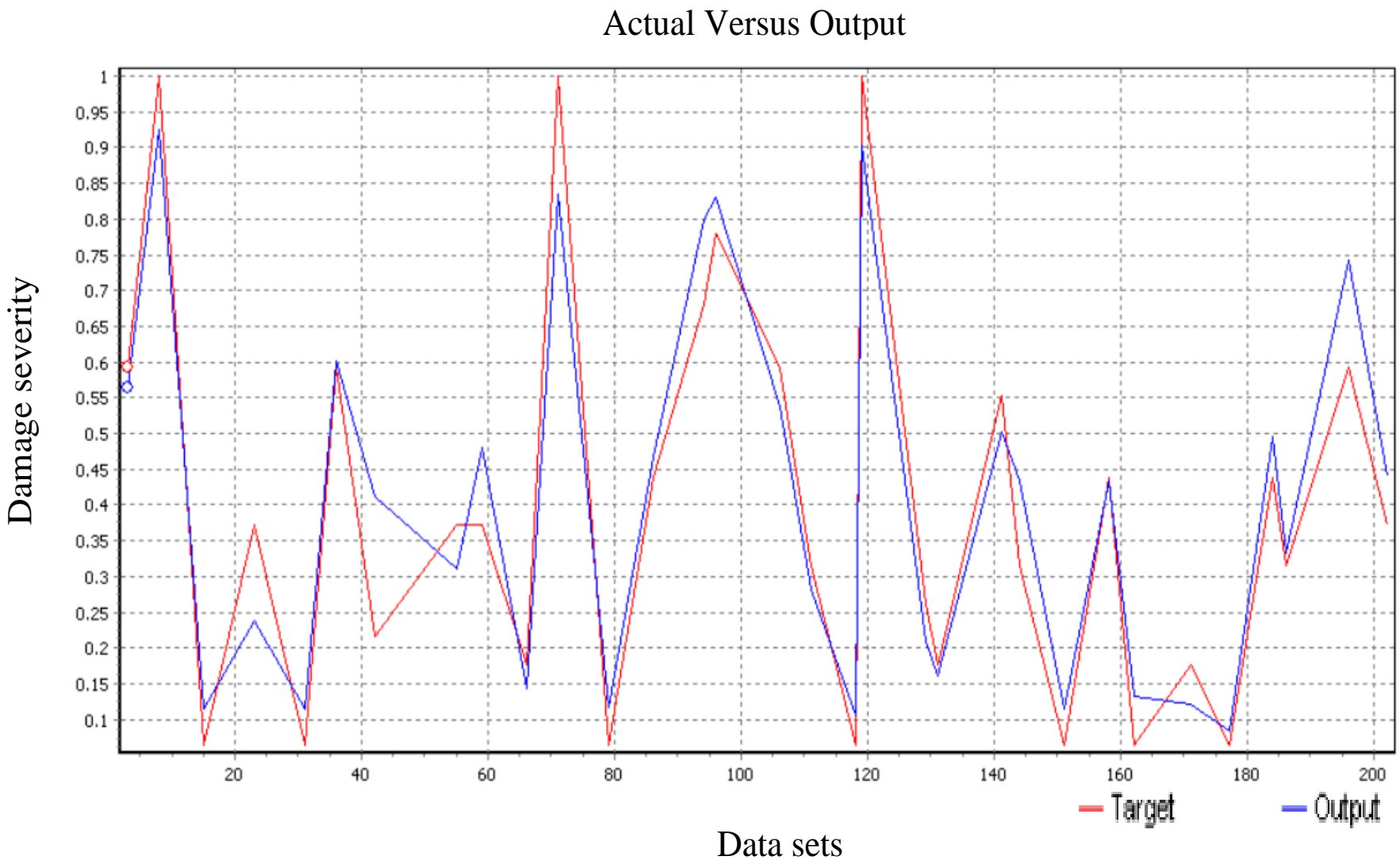

Figure 6. Comparison of damage severity predicted by ANN and experimental for testing sets. 


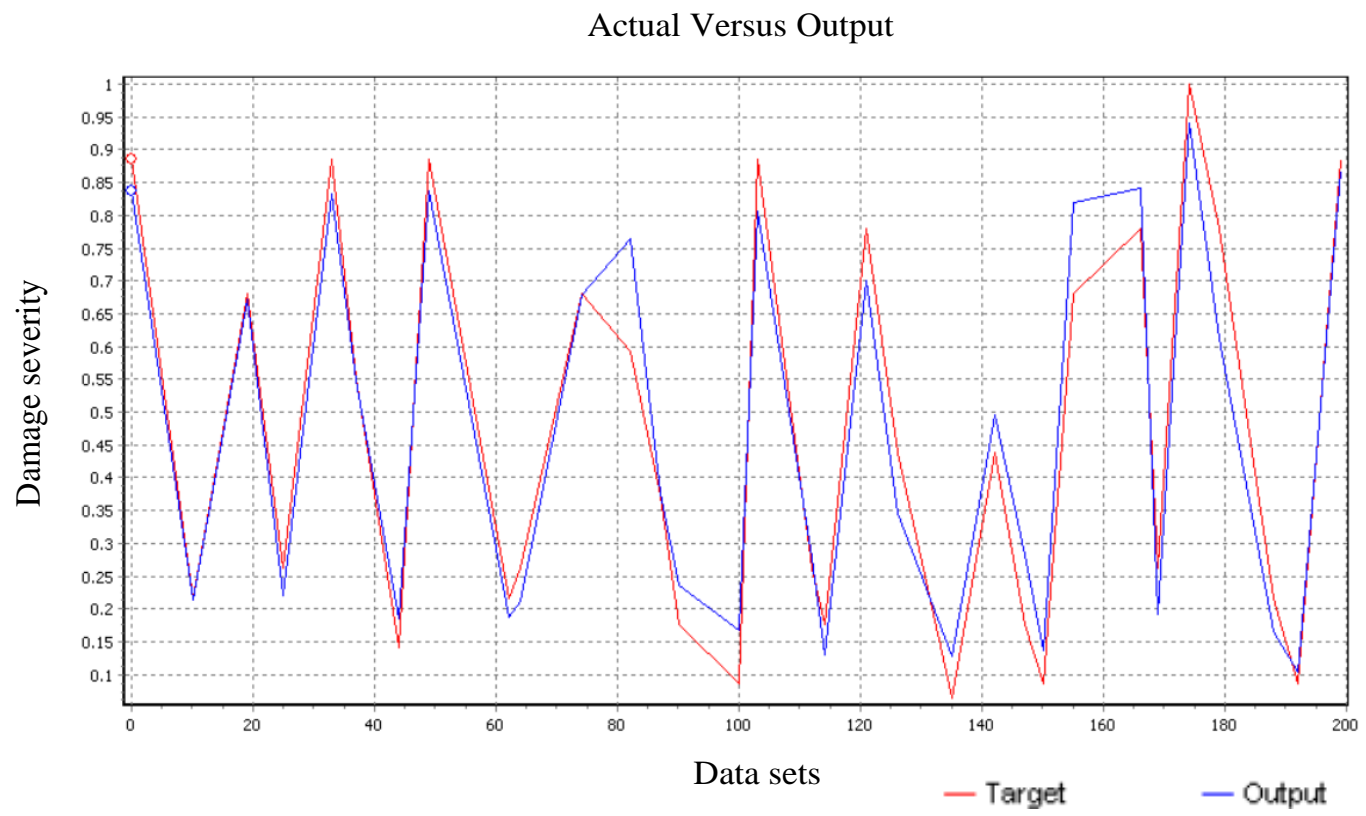

Figure 7. Comparison of damage severity predicted by ANN and experimental for validation sets

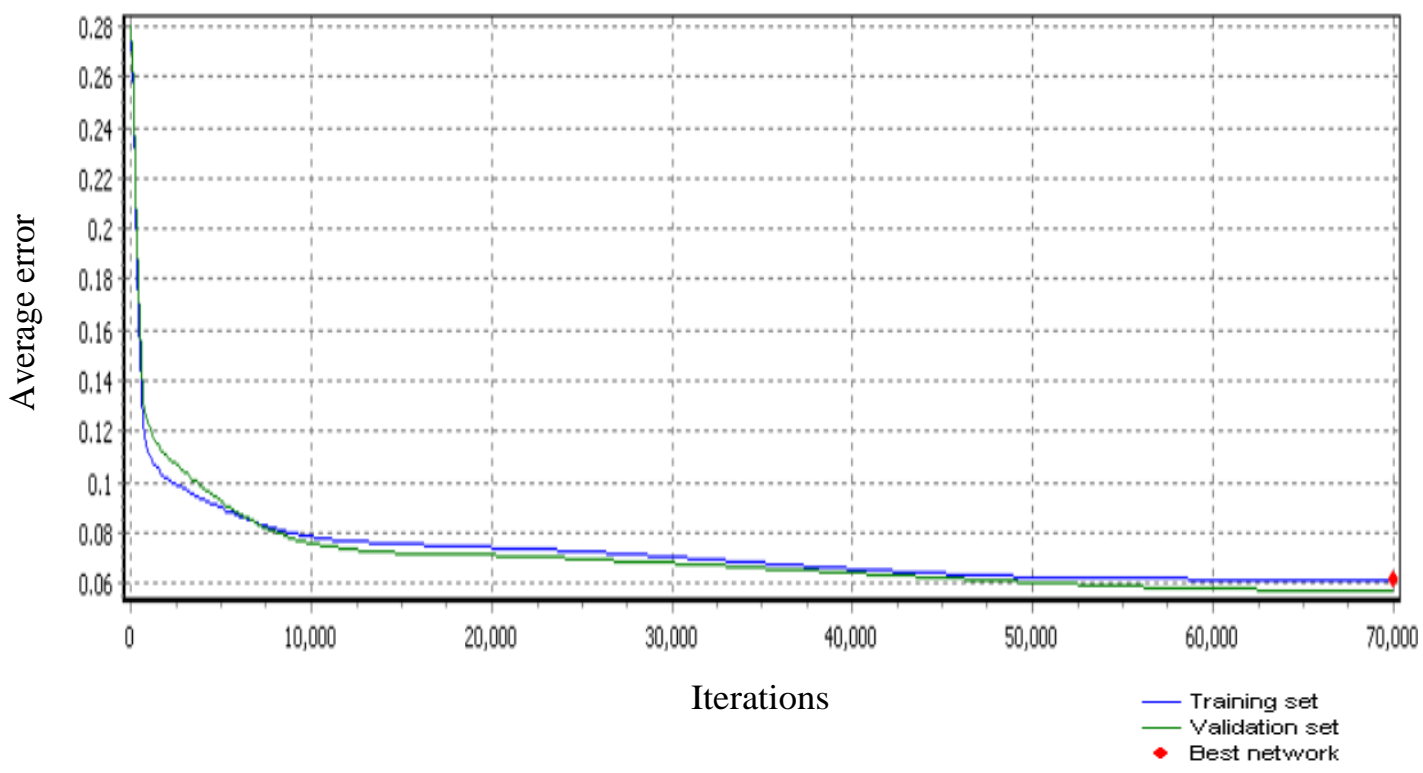

Figure 8. Comparison of average error of the training and validation sets versus number of iterations.

of data sets for different location of damage for training. It is a fact that the ANN can learn better and predict better when it is trained with more data.

\section{Validation phase}

The validation set is applied as a further check for the generalization of the neural network and to examine the accuracy of the selected architecture. The plot of predicted damage severity in validation sets against experimental data is as shown in Figure 7. It is clear from this figure that there is good agreement between the results predicted and target results. These results demonstrate that ANN was successful in training the relationship between the input and output data with the MSE of $7.79 \%$.

Comparison between average error of the training and validation data sets versus the number of iteration is depicted in Figure 8. As can be seen in this figure, the 


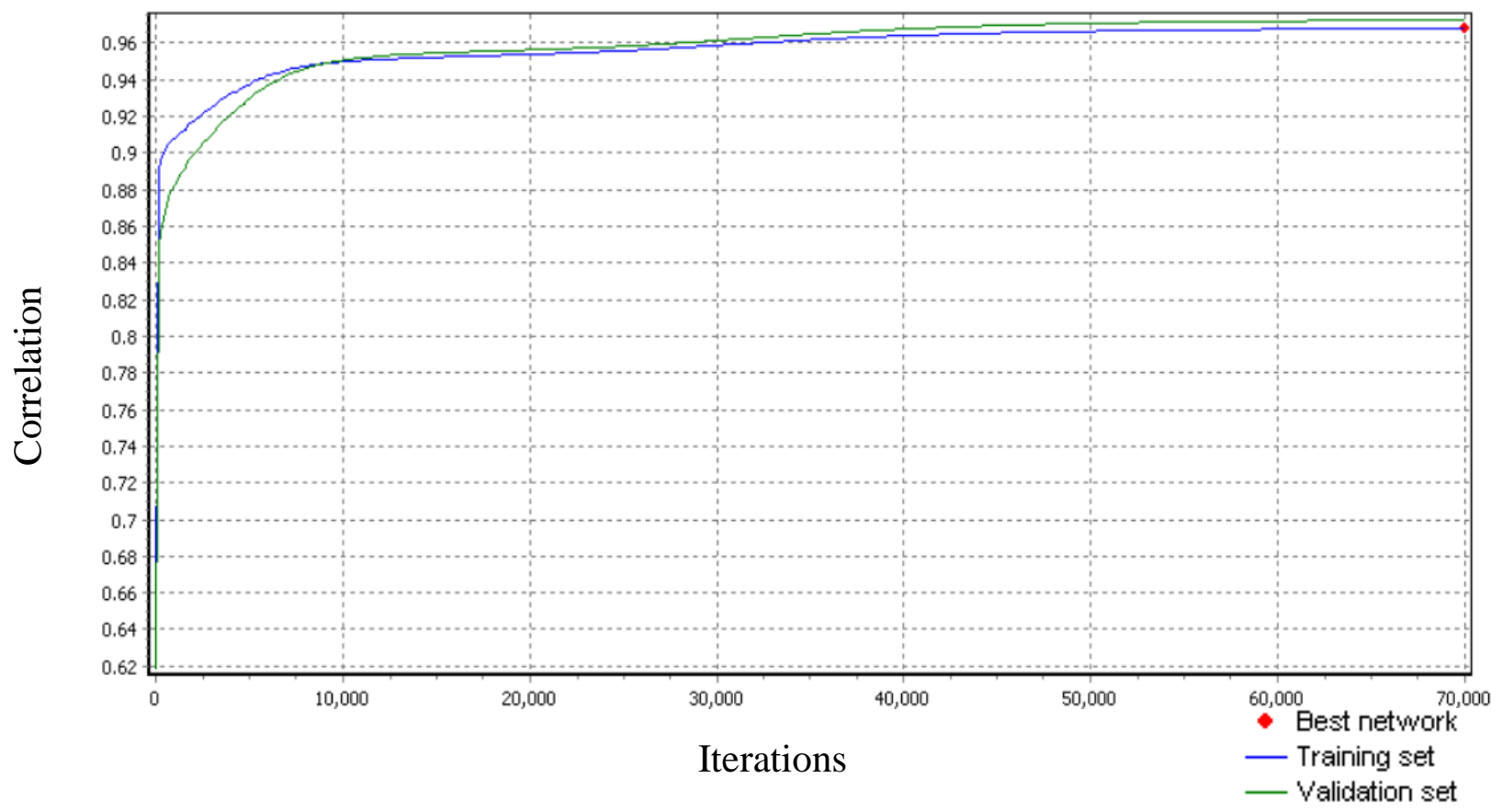

Figure 9. Correlation between training and validation data sets versus number of iterations.

results are fairly reasonable and the validation sets error has very similar characteristics and there is no over fitting. Figure 8 shows that the neural network model has an average error of about $5.62 \%$ for the training data and an error of about $7.79 \%$ for the validation data sets.

Also, the progress of the training was examined by plotting the correlations for training and validation data sets as shown in Figure 9. This figure also indicates that the results of training and validation are close and neural network was successful in learning the relationship between the different input parameters and damage severity as output.

\section{Conclusion}

In this research, the details of a study on using ANNs for prediction of damage severity in a model steel girder bridge are described. The dynamic tests conducted on the damaged and undamaged test structure showed that the reduction in stiffness during the damage lead to a reduction in natural frequencies for different modes. The experimentally obtained natural frequencies of the first five modes of the undamaged and damaged bridge model have been successfully applied as the training samples for the ANN.

According to results in this study, ANN could predict the damage severity with an error of 5.6, 6.25 and $7.79 \%$ for training, testing and validation, respectively. The feasibility of ANNs as a powerful tool for predicting the severity of damage in a structure is investigated.
Therefore, it is concluded that an ANN trained with just natural frequencies obtained from experimental modal analysis as inputs can very well be used to assess the severity of damage in a structure.

\section{REFERENCES}

Abdul RazakH, Choi FC (2001). The Effect of corrosion on the natural frequency and modal damping of reinforced concrete beams. J. Eng. Struct., 23(1): 1126-1133.

Chakraborty D (2005). Artificial neural network based delamination prediction in laminated composites. J. Mat. Design, 26: 1-7.

Chen HM, Tsai KH, Qi GZ, Yang JCS, Amini F (1995). Neural network for structure control. J. Comput. Civil Eng., 9(2): 168-175.

Fonseca ET, Vellasco PGS (2003). A path load parametric analysis using neural networks. J. Constructional Steel Res., 59: 251-267.

Ghaboussi J, Joghatatie A (1995). Active control of structures using neural networks. J. Eng. Mech., ASCE 121(4): 55-567.

Hakim SJS, Noorzaei J, Jaafar MS, Jameel M, Mohammadhassani M (2011). Application of Artificial Neural Networks to Predict Compressive strength of High Strength Concrete. Int. J. Phys. Sci., (IJPS). 6(5): 975-981.

Hola J, Schabowicz K (2005). Application of artificial neural networks to determine concrete compressive strength based on non-destructive tests. J. Civil Eng. Manag., 11(1): 23-32.

Ince R (2004). Prediction of fracture parameters of concrete by artificial neural networks. J. Eng. Fracture Mech., 71: 2143-2159.

Jeyasehar CA, Sumangala K (2006). Damage assessment of prestressed concrete beams using artificial neural network (ANN) approach. J. Computers Struct., 84: 1709-1718.

Kim YY, Kapania RK (2002). Neural networks for inverse problems in damage identification and optical imaging. 43rd AIAA/ASME/ASCE/AHS/ASC Structures. Structural Dynamics and Materials Conferences, Denver, Colorado.

Kirkegaard P, Rytter A (1994). Use of neural networks for damage assessment in a steel mast. Proceedings of the $12^{\text {th }}$ International 
Modal Analysis Conference. pp. 1128-1134.

Lee JJ, Lee JW, Yi JH, Yun CB, Jung HY (2005). Neural networksbased damage detection for bridges considering errors in baseline finite element models. J. Sound, Vibration. 280(3-5): 555-578.

Lee SC (2003). Prediction of concrete strength using artificial neural networks. J. Eng. Struct., 25: 849-857.

Mansour MY, Dicleli M, Lee YL, Zhang J (2004). Predicting the shear strength of reinforced concrete beams using artificial neural networks. J. Eng. Struct., 26: 781-799.

Rumelhart DE, Hinton GE, Williams RJ (1986). Learning representations by back propagation errors. J. Nature, 323: 533-536.

Sahin M, Shenoi RA (2003). Quantification and localization of damage in beam-like structures by using artificial neural networks with experimental validation. J. Eng. Struct., 25: 1785-1802.
Suh MW, Shim MB, Kim MY (2000).Crack identification using hybrid neuro-genetic technique. J. Sound Vibration, 234(4): 617-635.

Wu ZS, Xu B, Yokoyama K (2002). Decentralized parametric damage based on neural networks. J. Computer-Aided Civil Infrastruct. Eng., 17: $175-184$.

Xu B, Wu ZS, Yokoyama k (2002). A localized identification method with neural networks and its application to structural health monitoring. Structural Engineering. JSCE, 48A: 419-427. http://www.alyuda.com/index.html 